\title{
AUTOIMMUNITY
}

\section{SLE autoantibodies have lethal neurotoxic effects in female foetuses}

A subset of double-stranded DNA antibodies, present in $40 \%$ of patients with systemic lupus erythematosus (SLE), can induce apoptosis of neurons within the foetal brainstem, according to new mouse model data published in the Journal of Experimental Medicine. This neuronal apoptosis resulted in death of female foetuses.

The transmission of maternal antibodies across the placenta to the developing foetus is important for perinatal immunity. However, maternal autoantibodies that enter the foetal brain during the second trimester of pregnancy might have pathological effects and could cause learning difficulties in the children of women with SLE.

Betty Diamond and colleagues, authors of the new study, investigated foetal development in the presence of maternal SLE using pregnant mice immunized with a peptide that elicits the expression of anti-DNA antibodies. These antibodies cross-react with the activated $N$-methyl $D$-aspartate receptor (NMDAR) and cause excitotoxicity at high concentrations.

\section{4...maternal antibodies can kill foetuses in a gender- specific way... 77}

"Previously, we found impairments in some cortical tasks in the offspring exposed to antibody in utero", says Diamond, "but we also observed many more male offspring than female". The current study focused on brainstem, "because we needed to know why female foetuses were dying. Even during foetal development an intact brainstem is needed", she explains.

Binding of cross-reactive antibodies to the NR2A subunit of NMDAR induced apoptosis of neurons and resulted in the death of female foetuses. As there was no difference in the level of transmission of maternal autoantibodies to male and female foetal brains, the authors suggest that the gender-specific effects might be due to either increased expression of NR2A in female brainstem during a late stage of development or increased vulnerability of female foetal neurons to signalling through NR2A. They describe their findings as "very surprising" and conclude that maternal antibodies can kill foetuses in a gender-specific way.

Studies of other antibodies that might also affect foetal brain development, particularly in autism, are underway. Diamond is optimistic, "we might be able to predict risk to the foetus and even consider prevention strategies to protect the foetus from neurotoxic antibodies."

Ellen F. Carney

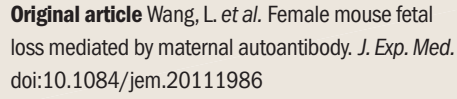

Original article Wang, L. et al. Female mouse fetal loss mediated by maternal autoantibody. J. Exp. Med. doi:10.1084/jem.20111986 